\title{
Bounded Positive Solutions for a Third Order Discrete Equation
}

\author{
Zeqing Liu, ${ }^{1}$ Ming Jia, ${ }^{2}$ Shin Min Kang, ${ }^{3}$ and Young Chel Kwun ${ }^{4}$ \\ ${ }^{1}$ Liaoning Normal University, Department of Mathematics, Dalian, Liaoning 116029, China \\ ${ }^{2}$ Dalian Hongwen Middle School, Dalian, Liaoning 116029, China \\ ${ }^{3}$ Gyeongsang National University, Department of Mathematics and RINS, \\ Jinju 660-701, Republic of Korea \\ ${ }^{4}$ Dong-A University, Department of Mathematics, Pusan 614-714, Republic of Korea \\ Correspondence should be addressed to Young Chel Kwun, yckwun@dau.ac.kr
}

Received 21 February 2012; Accepted 12 April 2012

Academic Editor: Josef Diblík

Copyright (C) 2012 Zeqing Liu et al. This is an open access article distributed under the Creative Commons Attribution License, which permits unrestricted use, distribution, and reproduction in any medium, provided the original work is properly cited.

This paper studies the following third order neutral delay discrete equation $\Delta\left(a_{n} \Delta^{2}\left(x_{n}+p_{n} x_{n-\tau}\right)\right)+$ $f\left(n, x_{n-d_{1 n}}, \ldots, x_{n-d_{l n}}\right)=g_{n}, n \geq n_{0}$, where $\tau, l \in \mathbb{N}, n_{0} \in \mathbb{N} \cup\{0\},\left\{a_{n}\right\}_{n \in \mathbb{N}_{n_{0}}},\left\{p_{n}\right\}_{n \in \mathbb{N}_{n_{0}}},\left\{g_{n}\right\}_{n \in \mathbb{N}_{n_{0}}}$ are real sequences with $a_{n} \neq 0$ for $n \geq n_{0},\left\{d_{i n}\right\}_{n \in \mathbb{N}_{n_{0}}} \subseteq \mathbb{Z}$ with $\lim _{n \rightarrow \infty}\left(n-d_{i n}\right)=+\infty$ for $i \in\{1,2, \ldots, l\}$ and $f \in C\left(\mathbb{N}_{n_{0}} \times \mathbb{R}^{l}, \mathbb{R}\right)$. By using a nonlinear alternative theorem of Leray-Schauder type, we get sufficient conditions which ensure the existence of bounded positive solutions for the equation. Three examples are given to illustrate the results obtained in this paper.

\section{Introduction and Preliminaries}

The oscillatory, nonoscillatory and asymptotic behaviors and existence of solutions for various difference equations have received more and more attentions in recent years. For details, we refer the reader to [1-11] and the references therein.

In 2005, M. Migda and J. Migda [10] studied the asymptotic behavior of solutions for the second order neutral difference equation

$$
\Delta^{2}\left(x_{n}+p x_{n-k}\right)+f\left(n, x_{n}\right)=0, \quad n \geq 1,
$$

where $p \in \mathbb{R}, k$ is a nonnegative integer and $f: \mathbb{N} \times \mathbb{R} \rightarrow \mathbb{R}$. In 2008, Cheng and Chu [7] established sufficientand necessary conditions of oscillation for the second order difference 
equation

$$
\Delta\left(r_{n-1} \Delta x_{n-1}\right)+p_{n} x_{n}^{\gamma}=0, \quad n \geq 1,
$$

where $\gamma$ is the quotient of two odd positive integers and $p_{n}, r_{n} \in(0,+\infty)$ for $n \in \mathbb{N}$. In 2000, Li et al. [9] gave several necessary and/or sufficient conditions of the existence of unbounded positive solution for the nonlinear difference equation

$$
\Delta\left(r_{n} \Delta x_{n}\right)+f\left(n, x_{n}\right)=0, \quad n \geq n_{0}
$$

where $n_{0}$ is a fixed nonnegative integer, $r: \mathbb{N}_{n_{0}} \rightarrow(0,+\infty)$ and $f: \mathbb{N}_{n_{0}} \times \mathbb{R} \rightarrow \mathbb{R}$. In 2003, using the Leray-Schauder's nonlinear alternative theorem, Agarwal et al. [1] presented the existence of nonoscillatory solutions for the discrete equation

$$
\Delta\left(a_{n} \Delta\left(x_{n}+p x_{n-\tau}\right)\right)+F\left(n+1, x_{n+1-\sigma}\right)=0, \quad n \geq 1,
$$

where $\tau, \sigma$ are fixed nonnegative integers, $p \in \mathbb{R}, a: \mathbb{N} \rightarrow(0,+\infty)$ and $F: \mathbb{N} \times(0,+\infty) \rightarrow$ $[0,+\infty)$ is continuous. In 1995, Yan and Liu [11] proved the existence of a bounded nonoscillatory solution for the third order difference equation

$$
\Delta^{3} x_{n}+f\left(n, x_{n}, x_{n-r}\right)=0, \quad n \geq n_{0}
$$

by utilizing the Schauder's fixed point theorem. In 2005, Andruch-Sobiło and Migda [2] studied the third order linear difference equations of neutral type

$$
\Delta^{3}\left(x_{n}-p_{n} x_{\sigma_{n}}\right) \pm q_{n} x_{\tau_{n}}=0, \quad n \geq n_{0}
$$

and obtained sufficient conditions under which all solutions of (1.6) are oscillatory.

The aim of this paper is to study the following third order neutral delay discrete equation

$$
\Delta\left(a_{n} \Delta^{2}\left(x_{n}+p_{n} x_{n-\tau}\right)\right)+f\left(n, x_{n-d_{1 n}}, \ldots, x_{n-d_{l n}}\right)=g_{n}, \quad n \geq n_{0}
$$

where $\tau, l \in \mathbb{N}, \varpi_{0} \in \mathbb{N} \cup\{0\},\left\{a_{n}\right\}_{n \in \mathbb{N}_{n_{0}}},\left\{p_{n}\right\}_{n \in \mathbb{N}_{n_{0}}},\left\{g_{n}\right\}_{n \in \mathbb{N}_{n_{0}}}$ are real sequences with $a_{n} \neq 0$ for $n \geq n_{0},\left\{d_{i n}\right\}_{n \in \mathbb{N}_{n_{0}}} \subseteq \mathbb{Z}$ with $\lim _{n \rightarrow \infty}\left(n-d_{i n}\right)=+\infty$ for $i \in\{1,2, \ldots, l\}$ and $f \in C\left(\mathbb{N}_{n_{0}} \times \mathbb{R}^{l}, \mathbb{R}\right)$. By making use of the Leray-Schauder's nonlinear alternative theorem, we establish the existence results of bounded positive solutions for (1.7), which extend substantially Theorem 2 in [11]. Three nontrivial examples are given to illustrate the superiority and applications of the results presented in this paper. 
Let us recall and introduce the below concepts, signs and lemmas. Let $\mathbb{R}, \mathbb{Z}$ and $\mathbb{N}$ denote the sets of all real numbers, integers and positive integers, respectively,

$$
\begin{gathered}
\mathbb{N}_{n_{0}}=\left\{n: n \in \mathbb{N} \text { with } n \geq n_{0}\right\}, \quad \mathbb{Z}_{\beta}=\{n: n \in \mathbb{Z} \text { with } n \geq \beta\}, \\
\beta=\min \left\{n_{0}-\tau, \inf \left\{n-d_{\text {in }}: 1 \leq i \leq l, n \in \mathbb{N}_{n_{0}}\right\}\right\}
\end{gathered}
$$

and $l_{\beta}^{\infty}$ stand for the Banach space of all bounded sequences on $\mathbb{Z}_{\beta}$ with norm

$$
\|x\|=\sup _{n \in \mathbb{Z}_{\beta}}\left|x_{n}\right| \quad \text { for } x=\left\{x_{n}\right\}_{n \in \mathbb{Z}_{\beta}} \in l_{\beta}^{\infty}
$$

For any constants $M>N>0$, put

$$
\begin{aligned}
E(N) & =\left\{x=\left\{x_{n}\right\}_{n \in \mathbb{Z}_{\beta}} \in l_{\beta}^{\infty}: x_{n} \geq N \text { for } n \in \mathbb{Z}_{\beta}\right\}, \\
U(M) & =\{x \in E(N):\|x\|<M\} .
\end{aligned}
$$

It is easy to verify that $E(N)$ is a nonempty closed convex subset of $l_{\beta}^{\infty}$ and $U(M)$ is a nonempty open subset of $E(N)$.

By a solution of (1.7), we mean a sequence $\left\{x_{n}\right\}_{n \in \mathbb{Z}_{\beta}}$ with a positive integer $T \geq \tau+|\beta|$ such that (1.7) holds for all $n \geq T$.

For any subset $U$ of a Banach space $X$, let $\bar{U}$ and $\partial U$ denote the closure and boundary of $U$ in $X$, respectively.

Lemma 1.1 (see [8]). A bounded, uniformly Cauchy subset $D$ of $l_{\beta}^{\infty}$ is relatively compact.

Lemma 1.2 (Leray-Schauder's Nonlinear Alternative Theorem [1]). Let E be a nonempty closed convex subset of a Banach space $X$ and $U$ be an open subset of $E$ with $p^{*} \in U$. Also $G: \bar{U} \rightarrow E$ is a continuous, condensing mapping with $G(\bar{U})$ bounded. Then either

$\left(\mathrm{A}_{1}\right)$ G has a fixed point in $\bar{U}$; or

$\left(\mathrm{A}_{2}\right)$ there are $x \in \partial U$ and $\lambda \in(0,1)$ with $x=(1-\lambda) p^{*}+\lambda G x$.

\section{Existence of Bounded Positive Solutions}

Now we investigate sufficient conditions of the existence of bounded positive solutions for (1.7) by using the Leray-Schauder's Nonlinear Alternative Theorem. 
Theorem 2.1. Assume that there exist constants $k_{0} \in \mathbb{N}_{n_{0}}$ and $M, N, \bar{p}$ and $\underline{p}$ satisfying

$$
\begin{gathered}
\sum_{s=k_{0}}^{\infty} \sum_{k=s}^{\infty} \frac{1}{\left|a_{k}\right|} \sum_{j=k}^{\infty} \sup \left\{\left|f\left(j, w_{1}, \ldots, w_{l}\right)\right|: w_{i} \in[N, M], 1 \leq i \leq l\right\}<+\infty ; \\
\sum_{s=k_{0}}^{\infty} \sum_{k=s}^{\infty} \frac{1}{\left|a_{k}\right|} \sum_{j=k}^{\infty}\left|g_{j}\right|<+\infty ; \\
0<N<(1-\underline{p}-\bar{p}) M, \quad \min \{\underline{p}, \bar{p}\} \geq 0, \quad \underline{p}+\bar{p}<1 \\
-\underline{p} \leq p_{n} \leq \bar{p}, \quad n \geq k_{0} .
\end{gathered}
$$

Then (1.7) possesses a bounded positive solution in $\overline{U(M)}$.

Proof. Let $L \in(\bar{p} M+N, M(1-\underline{p}))$. It follows from (2.1)-(2.3) that there exists a positive integer $T>1+\tau+k_{0}+|\beta|$ sufficiently large satisfying

$$
\begin{aligned}
& \sum_{s=T}^{\infty} \sum_{k=s}^{\infty} \frac{1}{\left|a_{k}\right|} \sum_{j=k}^{\infty}\left[\left|g_{j}\right|+\sup \left\{\left|f\left(j, w_{1}, \ldots, w_{l}\right)\right|: w_{i} \in[N, M], 1 \leq i \leq l\right\}\right] \\
& \quad<\min \{L-\bar{p} M-N, M(1-\underline{p})-L\}
\end{aligned}
$$

Choose $p^{*}=M-\varepsilon_{0}$ with $\varepsilon_{0} \in(0, \min \{L-\bar{p} M-N, M(1-\underline{p})-L\})$ and

$$
\begin{aligned}
& \sum_{s=T}^{\infty} \sum_{k=s}^{\infty} \frac{1}{\left|a_{k}\right|} \sum_{j=k}^{\infty}\left[\left|g_{j}\right|+\sup \left\{\left|f\left(j, w_{1}, \ldots, w_{l}\right)\right|: w_{i} \in[N, M], 1 \leq i \leq l\right\}\right] \\
& \quad \leq \min \{L-\bar{p} M-N, M(1-\underline{p})-L\}-\varepsilon_{0} .
\end{aligned}
$$

Note that

$$
M>M-\varepsilon_{0}=p^{*}>M-\min \{L-\bar{p} M-N, M(1-\underline{p})-L\} \geq N+M(1+\bar{p})-L>N
$$

which implies that $p^{*}=\left\{p^{*}\right\}_{n \in \mathbb{Z}_{\beta}} \in U(M)$. Define two mappings $A_{L}, B_{L}: \overline{U(M)} \rightarrow l_{\beta}^{\infty}$ 
by

$$
\begin{aligned}
& A_{L} x_{n}= \begin{cases}L-p_{n} x_{n-\tau}, & n \geq T+1 \\
L-p_{T} x_{T}, & \beta \leq n \leq T ;\end{cases} \\
& B_{L} x_{n}= \begin{cases}-\sum_{s=T}^{n-1} \sum_{k=s}^{\infty} \frac{1}{a_{k}} \sum_{j=k}^{\infty}\left[-g_{j}+f\left(j, x_{j-d_{1 j}}, \ldots, x_{j-d_{l j}}\right)\right], & n \geq T+1 \\
0, & \beta \leq n \leq T\end{cases}
\end{aligned}
$$

for all $x=\left\{x_{n}\right\}_{n \in \mathbb{Z}_{\beta}} \in \overline{U(M)}$.

We now show that

$$
D_{L}=A_{L}+B_{L}: \overline{U(M)} \longrightarrow E(N)
$$

For each $x=\left\{x_{n}\right\}_{n \in \mathbb{Z}_{\beta}} \in \overline{U(M)}$, by (2.4)-(2.9), we have

$$
\begin{aligned}
A_{L} x_{n}+B_{L} x_{n}= & L-p_{n} x_{n-\tau}-\sum_{s=T}^{n-1} \sum_{k=s}^{\infty} \frac{1}{a_{k}} \sum_{j=k}^{\infty}\left[-g_{j}+f\left(j, x_{j-d_{1 j}}, \ldots, x_{j-d_{l j}}\right)\right] \\
& \geq L-\bar{p} M-\sum_{s=T}^{n-1} \sum_{k=s}^{\infty} \frac{1}{\left|a_{k}\right|} \sum_{j=k}^{\infty}\left[\left|g_{j}\right|+\left|f\left(j, x_{j-d_{1 j}}, \ldots, x_{j-d_{l j}}\right)\right|\right] \\
& \geq L-\bar{p} M-\min \{L-\bar{p} M-N, M(1-\underline{p})-L\}+\varepsilon_{0} \\
& \geq N+\varepsilon_{0} \\
& >N, \quad n \geq T+1 \\
A_{L} x_{n} & +B_{L} x_{n}=L-p_{T} x_{T} \geq L-\bar{p} M>N, \quad \beta \leq n \leq T,
\end{aligned}
$$

We next assert that

$$
B_{L}: \overline{U(M)} \longrightarrow l_{\beta}^{\infty} \text { is a continuous, compact mapping. }
$$

Let $\left\{x^{\alpha}\right\}_{\alpha \in \mathbb{N}} \subseteq \overline{U(M)}$ be an arbitrary sequence and $x^{0} \in l_{\beta}^{\infty}$ with

$$
\left\|x^{\alpha}-x^{0}\right\| \longrightarrow 0 \quad \text { as } \alpha \longrightarrow \infty
$$

Since $\overline{U(M)}$ is closed, it follows that $x^{0} \in \overline{U(M)}$. Given $\varepsilon>0$. Using (2.1), (2.13) and the 
continuity of $f$, we infer that there exists $T^{* *}, T^{*} \in \mathbb{N}$ with $T^{*}>T+1$ satisfying

$$
\begin{gathered}
\sum_{s=T^{*}}^{\infty} \sum_{k=s}^{\infty} \frac{1}{\left|a_{k}\right|} \sum_{j=k}^{\infty} \sup \left\{\left|f\left(j, w_{1}, \ldots, w_{l}\right)\right|: w_{i} \in[N, M], 1 \leq i \leq l\right\}<\frac{\varepsilon}{16} \\
\sum_{k=T^{*}}^{\infty} \frac{1}{\left|a_{k}\right|} \sum_{j=k}^{\infty} \sup \left\{\left|f\left(j, w_{1}, \ldots, w_{l}\right)\right|: w_{i} \in[N, M], 1 \leq i \leq l\right\}<\frac{\varepsilon}{16\left(T^{*}-T\right)} \\
\sum_{s=T}^{T^{*}-1} \sum_{k=s}^{T^{*}-1} \frac{1}{\left|a_{k}\right|} \sum_{j=T^{*}}^{\infty} \sup \left\{\left|f\left(j, w_{1}, \ldots, w_{l}\right)\right|: w_{i} \in[N, M], 1 \leq i \leq l\right\}<\frac{\varepsilon}{16} \\
\sum_{s=T}^{T^{*}-1} \sum_{k=s}^{T^{*}-1} \frac{1}{\left|a_{k}\right|} \sum_{j=k}^{T^{*}-1}\left|f\left(j, x_{j-d_{1 j}}^{\alpha}, \ldots, x_{j-d_{l j}}^{\alpha}\right)-f\left(j, x_{j-d_{1 j}}^{0}, \ldots, x_{j-d_{l j}}^{0}\right)\right|<\frac{\varepsilon}{2}, \quad \alpha \geq T^{* *}
\end{gathered}
$$

Combining (2.9) and (2.14)-(2.17), we conclude that

$$
\begin{aligned}
& \left\|B_{L} x^{\alpha}-B_{L} x^{0}\right\| \\
& =\max \left\{\sup _{\beta \leq n \leq T}\left|B_{L} x_{n}^{\alpha}-B_{L} x_{n}^{0}\right|, \sup _{n \geq T+1}\left|B_{L} x_{n}^{\alpha}-B_{L} x_{n}^{0}\right|\right\} \\
& \leq \max \left\{0, \sup _{n \geq T+1} \sum_{S=T}^{n-1} \sum_{k=S}^{\infty} \frac{1}{\left|a_{k}\right|} \sum_{j=k}^{\infty}\left|f\left(j, x_{j-d_{1 j}}^{\alpha}, \ldots, x_{j-d_{l j}}^{\alpha}\right)-f\left(j, x_{j-d_{1 j}}^{0}, \ldots, x_{j-d_{l j}}^{0}\right)\right|\right\} \\
& \leq \sum_{s=T}^{T^{*}-1} \sum_{k=S}^{\infty} \frac{1}{\left|a_{k}\right|} \sum_{j=k}^{\infty}\left|f\left(j, x_{j-d_{1 j}}^{\alpha}, \ldots, x_{j-d_{l j}}^{\alpha}\right)-f\left(j, x_{j-d_{1 j}}^{0}, \ldots, x_{j-d_{l j}}^{0}\right)\right| \\
& +\sum_{s=T^{*}}^{\infty} \sum_{k=s}^{\infty} \frac{1}{\left|a_{k}\right|} \sum_{j=k}^{\infty}\left[\left|f\left(j, x_{j-d_{1 j}}^{\alpha}, \ldots, x_{j-d_{l j}}^{\alpha}\right)\right|+\left|f\left(j, x_{j-d_{1 j}}^{0}, \ldots, x_{j-d_{l j}}^{0}\right)\right|\right] \\
& \leq \sum_{s=T}^{T^{*}-1} \sum_{k=S}^{T^{*}-1} \frac{1}{\left|a_{k}\right|} \sum_{j=k}^{\infty}\left|f\left(j, x_{j-d_{1 j}}^{\alpha}, \ldots, x_{j-d_{l j}}^{\alpha}\right)-f\left(j, x_{j-d_{1 j}}^{0}, \ldots, x_{j-d_{l j}}^{0}\right)\right| \\
& +\sum_{s=T}^{T^{*}-1} \sum_{k=T^{*}}^{\infty} \frac{1}{\left|a_{k}\right|} \sum_{j=k}^{\infty}\left|f\left(j, x_{j-d_{1 j}}^{\alpha}, \ldots, x_{j-d_{l j}}^{\alpha}\right)\right|+\left|f\left(j, x_{j-d_{1 j^{\prime}}}^{0}, \ldots, x_{j-d_{l j}}^{0}\right)\right| \\
& +2 \sum_{s=T^{*}}^{\infty} \sum_{k=s}^{\infty} \frac{1}{\left|a_{k}\right|} \sum_{j=k}^{\infty} \sup \left\{\left|f\left(j, w_{1}, \ldots, w_{l}\right)\right|: w_{i} \in[N, M], \quad 1 \leq i \leq l\right\} \\
& \leq \sum_{s=T}^{T^{*}-1} \sum_{k=S}^{T^{*}-1} \frac{1}{\left|a_{k}\right|} \sum_{j=k}^{T^{*}-1}\left|f\left(j, x_{j-d_{1 j}}^{\alpha}, \ldots, x_{j-d_{l j}}^{\alpha}\right)-f\left(j, x_{j-d_{1 j}}^{0}, \ldots, x_{j-d_{l j}}^{0}\right)\right| \\
& +\sum_{s=T}^{T^{*}-1} \sum_{k=S}^{T^{*}-1} \frac{1}{\left|a_{k}\right|} \sum_{j=T^{*}}^{\infty}\left[\left|f\left(j, x_{j-d_{1 j}}^{\alpha}, \ldots, x_{j-d_{l j}}^{\alpha}\right)\right|+\left|f\left(j, x_{j-d_{1 j}}^{0}, \ldots, x_{j-d_{l j}}^{0}\right)\right|\right] \\
& +2\left(T^{*}-T\right) \sum_{k=T^{*}}^{\infty} \frac{1}{\left|a_{k}\right|} \sum_{j=k}^{\infty} \sup \left\{\left|f\left(j, w_{1}, \ldots, w_{l}\right)\right|: w_{i} \in[N, M], 1 \leq i \leq l\right\}+2 \cdot \frac{\varepsilon}{16} \\
& <\frac{\varepsilon}{2}+2 \cdot \frac{\varepsilon}{16}+2\left(T^{*}-T\right) \cdot \frac{\varepsilon}{16\left(T^{*}-T\right)}+\frac{\varepsilon}{8} \\
& <\varepsilon, \quad \alpha \geq T^{* *},
\end{aligned}
$$


which means that $B_{L}$ is continuous in $\overline{U(M)}$. On the other hand, in light of (2.6) and (2.9), we get that for each $x=\left\{x_{n}\right\}_{n \in \mathbb{Z}_{\beta}} \in \overline{U(M)}$

$$
\begin{aligned}
\left\|B_{L} x\right\| & =\max \left\{\sup _{\beta \leq n \leq T}\left|B_{L} x_{n}\right|, \sup _{n \geq T+1}\left|B_{L} x_{n}\right|\right\} \\
& \leq \sup _{n \geq T+1} \sum_{s=T}^{n-1} \sum_{k=s}^{\infty} \frac{1}{\left|a_{k}\right|} \sum_{j=k}^{\infty}\left[\left|g_{j}\right|+\left|f\left(j, x_{j-d_{1 j}}, \ldots, x_{j-d_{l j}}\right)\right|\right] \\
& \leq \sum_{s=T}^{\infty} \sum_{k=s}^{\infty} \frac{1}{\left|a_{k}\right|} \sum_{j=k}^{\infty}\left[\left|g_{j}\right|+\sup \left\{\left|f\left(j, w_{1}, \ldots, w_{l}\right)\right|: w_{i} \in[N, M], 1 \leq i \leq l\right\}\right] \\
& \leq \min \{L-\bar{p} M-N, M(1-\underline{p})-L\}-\varepsilon_{0} \\
& \leq M,
\end{aligned}
$$

which yields that $B_{L}(\overline{U(M)})$ is a bounded subset of $l_{\beta}^{\infty}$. By virtue of (2.1) and (2.2), we deduce that for any $\varepsilon>0$, there exists $T_{0}>T$ satisfying

$$
\sum_{s=T_{0}}^{\infty} \sum_{k=s}^{\infty} \frac{1}{\left|a_{k}\right|} \sum_{j=k}^{\infty}\left[\left|g_{j}\right|+\sup \left\{\left|f\left(j, w_{1}, \ldots, w_{l}\right)\right|: w_{i} \in[N, M], 1 \leq i \leq l\right\}\right]<\varepsilon,
$$

which together with (2.9) gives that for any $x=\left\{x_{n}\right\}_{n \in \mathbb{Z}_{\beta}} \in \overline{U(M)}$

$$
\begin{aligned}
& \left|B_{L} x_{n}-B_{L} x_{m}\right| \\
& =\left|\sum_{s=T}^{n-1} \sum_{k=s}^{\infty} \frac{1}{a_{k}} \sum_{j=k}^{\infty}\left[-g_{j}+f\left(j, x_{j-d_{1 j}}, \ldots, x_{j-d_{l j}}\right)\right]-\sum_{s=T}^{m-1} \sum_{k=s}^{\infty} \frac{1}{a_{k}} \sum_{j=k}^{\infty}\left[-g_{j}+f\left(j, x_{j-d_{1 j}}, \ldots, x_{j-d_{l j}}\right)\right]\right| \\
& \leq \sum_{s=n}^{m-1} \sum_{k=s}^{\infty} \frac{1}{\left|a_{k}\right|} \sum_{j=k}^{\infty}\left[\left|g_{j}\right|+\left|f\left(j, x_{j-d_{1 j}}, \ldots, x_{j-d_{l j}}\right)\right|\right] \\
& <\sum_{s=T_{0}}^{\infty} \sum_{k=s}^{\infty} \frac{1}{\left|a_{k}\right|} \sum_{j=k}^{\infty}\left[\left|g_{j}\right|+\sup \left\{\left|f\left(j, w_{1}, \ldots, w_{l}\right)\right|: w_{i} \in[N, M], 1 \leq i \leq l\right\}\right] \\
& <\varepsilon, \quad m>n \geq T_{0},
\end{aligned}
$$

which means that $B_{L}(\overline{U(M)})$ is uniformly Cauchy. Thus Lemma 1.1 ensures that $B_{L}(\overline{U(M)})$ is a relatively compact subset of $l_{\beta}^{\infty}$.

Let $x=\left\{x_{n}\right\}_{n \in \mathbb{Z}_{\beta}}, y=\left\{y_{n}\right\}_{n \in \mathbb{Z}_{\beta}} \in \overline{U(M)}$. In view of (2.3), (2.4) and (2.8), we know that

$$
\begin{array}{ll}
\left|A_{L} x_{n}-A_{L} y_{n}\right|=\left|L-p_{n} x_{n-\tau}-L+p_{n} y_{n-\tau}\right| \leq\left|p_{n}\right|\|x-y\| \leq(\underline{p}+\bar{p})\|x-y\|, & n \geq T+1, \\
\left|A_{L} x_{n}-A_{L} y_{n}\right|=\left|L-p_{T} x_{T-\tau}-L+p_{T} y_{T-\tau}\right| \leq\left|p_{T}\right|\|x-y\| \leq(\underline{p}+\bar{p})\|x-y\|, & \beta \leq n \leq T,
\end{array}
$$


which implies that

$$
\|A x-A y\| \leq(\underline{p}+\bar{p})\|x-y\|
$$

which together with (2.10) and (2.12) guarantees that $D_{L}: \overline{U(M)} \rightarrow E(N)$ is a continuous, condensing mapping.

In order to show the existence of a fixed point of $D_{L}$, we need to prove that $\left(\mathrm{A}_{2}\right)$ in Lemma 1.2 does not hold. Otherwise there exist $x=\left\{x_{n}\right\}_{n \in \mathbb{Z}_{\beta}} \in \partial U(M)$ and $\lambda \in(0,1)$ such that $x=(1-\lambda) p^{*}+\lambda D_{L} x$. Let

$$
\begin{gathered}
S_{1}=\left\{x \in l_{\beta}^{\infty}: N \leq x_{n} \leq M, \forall n \geq \beta,\|x\|=M\right\}, \\
S_{2}=\left\{x \in l_{\beta}^{\infty}: N \leq x_{n} \leq M, \forall n \geq \beta, \text { and there exists } n^{*} \geq \beta \text { satisfying } x_{n^{*}}=N\right\} .
\end{gathered}
$$

It is easy to verify that $\partial U(M)=S_{1} \cup S_{2}$. Now we have to discuss two possible cases as follows:

Case 1. Let $x \in S_{1}$. It follows from (2.3), (2.4), (2.8) and (2.9) that

$$
\begin{aligned}
x_{n} & =(1-\lambda) p^{*}+\lambda\left[A_{L} x_{n}+B_{L} x_{n}\right] \\
& =(1-\lambda) p^{*}+\lambda\left[L-p_{n} x_{n-\tau}-\sum_{s=T}^{n-1} \sum_{k=s}^{\infty} \frac{1}{a_{k}} \sum_{j=k}^{\infty}\left[-g_{j}+f\left(j, x_{j-d_{1 j}}, \ldots, x_{j-d_{l j}}\right)\right]\right] \\
& \leq(1-\lambda) p^{*}+\lambda\left[L+\underline{p} M+\sum_{s=T} \sum_{k=s}^{n-1} \frac{1}{\left|a_{k}\right|} \sum_{j=k}^{\infty}\left[\left|g_{j}\right|+\left|f\left(j, x_{j-d_{1 j}}, \ldots, x_{j-d_{l j}}\right)\right|\right]\right] \\
& \leq(1-\lambda)\left(M-\varepsilon_{0}\right)+\lambda\left[L+\underline{p} M+\min \{L-\bar{p} M-N, M(1-\underline{p})-L\}-\varepsilon_{0}\right] \\
& \leq(1-\lambda)\left(M-\varepsilon_{0}\right)+\lambda\left(M-\varepsilon_{0}\right) \\
& =M-\varepsilon_{0}, \quad n \geq T+1, \\
x_{n} & =(1-\lambda) p^{*}+\lambda\left[L-p_{T} x_{T}\right] \leq(1-\lambda) p^{*}+\lambda(L+\underline{p} M) \\
& \leq(1-\lambda)\left(M-\varepsilon_{0}\right)+\lambda\left(M-\varepsilon_{0}\right)=M-\varepsilon_{0}, \quad \beta \leq n \leq T,
\end{aligned}
$$

which yield that

$$
M=\|x\| \leq M-\varepsilon_{0}<M,
$$

which is a contradiction; 
Case 2. Let $x \in S_{2}$. If $n^{*} \geq T+1$, by (2.3), (2.4), (2.8) and (2.9), we deduce that

$$
\begin{aligned}
N & =x_{n^{*}}=(1-\lambda) p^{*}+\lambda\left[A_{L} x_{n^{*}}+B_{L} x_{n^{*}}\right] \\
& =(1-\lambda) p^{*}+\lambda\left[L-p_{n^{*}} x_{n^{*}-\tau}-\sum_{s=T}^{n^{*}-1} \sum_{k=s}^{\infty} \frac{1}{a_{k}} \sum_{j=k}^{\infty}\left[-g_{j}+f\left(j, x_{j-d_{1 j}}, \ldots, x_{j-d_{l j}}\right)\right]\right] \\
& \geq(1-\lambda)\left(M-\varepsilon_{0}\right)+\lambda\left[L-\bar{p} M-\sum_{s=T}^{n^{*}-1} \sum_{k=s}^{\infty} \frac{1}{\left|a_{k}\right|} \sum_{j=k}^{\infty}\left[\left|g_{j}\right|+\left|f\left(j, x_{j-d_{1 j}}, \ldots, x_{j-d_{l j}}\right)\right|\right]\right] \\
& >(1-\lambda) N+\lambda\left[L-\bar{p} M-\min \{L-\bar{p} M-N, \quad M(1-\underline{p})-L\}+\varepsilon_{0}\right] \\
& \geq(1-\lambda) N+\lambda\left(N+\varepsilon_{0}\right) \\
& =N+\varepsilon_{0} \\
& >N
\end{aligned}
$$

which is impossible; if $n^{*} \leq T$, by (2.3), (2.4), (2.8) and (2.9), we arrive at

$$
\begin{aligned}
N & =x_{n^{*}}=(1-\lambda) p^{*}+\lambda\left[L-p_{T} x_{T}\right] \geq(1-\lambda) p^{*}+\lambda(L-\bar{p} M) \\
& \geq(1-\lambda) N+\lambda\left(N+\varepsilon_{0}\right)=N+\varepsilon_{0} \\
& >N
\end{aligned}
$$

which is absurd.

Consequently Lemma 1.2 ensures that there is $x=\left\{x_{n}\right\}_{n \in \mathbb{Z}_{\beta}} \in \overline{U(M)}$ such that $D_{L} x=$ $A_{L} x+B_{L} x=x$, which is a bounded positive solution of (1.7). This completes the proof.

Remark 2.2. Under the conditions of Theorem 2.1 we prove also that (1.7) has uncountably many bounded positive solutions in $\overline{U(M)}$.

In fact, as in the proof of Theorem 2.1, for any different $L_{1}, L_{2} \in(\bar{p} M+N, M(1-p))$ we conclude that for each $r \in\{1,2\}$, there exist a constant $T_{r}>1+\tau+k_{0}+|\beta|$ and two mappings $A_{r}, B_{r}: \overline{U(M)} \rightarrow l_{\beta}^{\infty}$ satisfying (2.6)-(2.9) and

$$
\sum_{s=\min \left\{T_{1}, T_{2}\right\}}^{\infty} \sum_{k=s}^{\infty} \frac{1}{\left|a_{k}\right|} \sum_{j=k}^{\infty}\left[\left|g_{j}\right|+\sup \left\{\left|f\left(j, w_{1}, \ldots, w_{l}\right)\right|: w_{i} \in[N, M], 1 \leq i \leq l\right\}\right] \leq \frac{\left|L_{1}-L_{2}\right|}{4}
$$

where $T, L, A_{L}$ and $B_{L}$ are replaced by $T_{r}, r, A_{r}$ and $B_{r}$, respectively, and $A_{r}+B_{r}$ has a fixed point $z^{r}=\left\{z_{n}^{r}\right\}_{n \in \mathbb{Z}_{\beta}} \in \overline{U(M)}$, which is a bounded positive solution of (1.7). In order to prove 
that (1.7) possesses uncountably many bounded positive solutions in $\overline{U(M)}$, we need only to prove that $z^{1} \neq z^{2}$. It follows from (2.8), (2.9) and (2.30) that for $n \geq \min \left\{T_{1}, T_{2}\right\}$

$$
\begin{aligned}
\left|z_{n}^{1}-z_{n}^{2}\right|= & \mid L_{1}-p_{n} z_{n-\tau}^{1}-\sum_{s=T_{1}}^{n-1} \sum_{k=s}^{\infty} \frac{1}{a_{k}} \sum_{j=k}^{\infty}\left[-g_{j}+f\left(j, z_{j-d_{1 j}}^{1}, \ldots, z_{j-d_{l j}}^{1}\right)\right] \\
& -L_{2}+p_{n} z_{\mathrm{n}-\tau}^{2}+\sum_{s=T_{2}}^{n-1} \sum_{k=s}^{\infty} \frac{1}{a_{k}} \sum_{j=k}^{\infty}\left[-g_{j}+f\left(j, z_{j-d_{1 j}}^{2}, \ldots, z_{j-d_{l j}}^{2}\right)\right] \mid \\
\geq & \left|L_{1}-L_{2}\right|-(\underline{p}+\bar{p})\left\|z^{1}-z^{2}\right\| \\
& -2 \sum_{s=\min \left\{T_{1}, T_{2}\right\}}^{\infty} \sum_{k=s}^{\infty} \frac{1}{\left|a_{k}\right|} \sum_{j=k}^{\infty}\left[\left|g_{j}\right|+\sup \left\{\left|f\left(j, w_{1}, \ldots, w_{l}\right)\right|: w_{i} \in[N, M], 1 \leq i \leq l\right\}\right] \\
\geq & \left|L_{1}-L_{2}\right|-(\underline{p}+\bar{p})\left\|z^{1}-z^{2}\right\|-\frac{\left|L_{1}-L_{2}\right|}{2},
\end{aligned}
$$

which implies that

$$
\left\|z^{1}-z^{2}\right\| \geq \frac{\left|L_{1}-L_{2}\right|}{2(1+\underline{p}+\bar{p})}>0,
$$

which yields that $z^{1} \neq z^{2}$.

Remark 2.3. If either $\underline{p}=0$ or $\bar{p}=0$, then Theorem 2.1 reduces to the below results, respectively.

Theorem 2.4. Assume that there exist constants $k_{0} \in \mathbb{N}_{n_{0}}$ and $M, N$ and $\bar{p}$ satisfying (2.1), (2.2) and

$$
0<N<(1-\bar{p}) M, \quad 0 \leq p_{n} \leq \bar{p}<1, n \geq k_{0} .
$$

Then (1.7) possesses a bounded positive solution in $\overline{U(M)}$.

Theorem 2.5. Assume that there exist constants $k_{0} \in \mathbb{N}_{n_{0}}$ and $M, N$ and $\underline{p}$ satisfying (2.1), (2.2) and

$$
0<N<(1-\underline{p}) M, \quad-1<-\underline{p} \leq p_{n} \leq 0, \quad n \geq k_{0} .
$$

Then (1.7) possesses a bounded positive solution in $\overline{U(M)}$.

Remark 2.6. Theorems 2.1-2.3 include Theorem 2 in [11] as special cases. Examples 3.1-3.3 in Section 3 explain that Theorems 2.1-2.3 are genuine generalizations of Theorem 2 in [11]. 


\section{Examples and Applications}

Now we construct three nontrivial examples to explain the superiority and applications of Theorems 2.1-2.3, respectively.

Example 3.1. Consider the third order neutral delay discrete equation

$$
\Delta\left((-1)^{n} n^{3} \Delta^{2}\left(x_{n}+\frac{n \sin \left(n^{2}\right)}{3 n+1} x_{n-\tau}\right)\right)+\frac{n^{3} x_{n^{2}-2}-\sqrt{n} x_{n^{3}-6}^{3}}{n^{5}+5 n+n x_{2 n+5}^{2}}=\frac{\cos \left(n \ln \left(n^{2}+1\right)\right)}{\sqrt{n^{3}+1}}, \quad n \geq 1,
$$

where $\tau \in \mathbb{N}$ is fixed. Let $l=3, n_{0}=1, k_{0}=2, \underline{p}=1 / 2, \bar{p}=1 / 3, M=7, N=1$,

$$
\begin{aligned}
& a_{n}=(-1)^{n} n^{3}, \quad p_{n}=\frac{n \sin \left(n^{2}\right)}{3 n+1}, \\
& g_{n}=\frac{\cos \left(n \ln \left(n^{2}+1\right)\right)}{\sqrt{n^{3}+1}}, \quad d_{1 n}=-n^{2}+n+2, \\
& d_{3 n}=-n-5, \quad f(n, u, v, w)=\frac{n^{3} u-\sqrt{n} v^{3}}{n^{5}+5 n+n w^{2}}, \quad \forall(n, u, v, w) \in \mathbb{N} \times \mathbb{R}^{3} .
\end{aligned}
$$

It is easy to verify that (2.1)-(2.4) hold. It follows from Theorem 2.1 that (3.1) has a bounded positive solution in $\overline{U(M)}$. However Theorem 2 in [11] is useless for (3.1).

Example 3.2. Consider the third order neutral delay discrete equation

$$
\Delta\left((n+1)^{2} \ln ^{3}(n+2) \Delta^{2}\left(x_{n}+\frac{3 n-4}{4 n+2} x_{n-\tau}\right)\right)+\frac{x_{n(n+1) / 2}^{2} x_{n(n-1) / 2}^{3}}{\sqrt{n^{3}+1}}=\frac{(-1)^{n}}{n^{2}}, \quad n \geq 1,
$$

where $\tau \in \mathbb{N}$ is fixed. Let $l=2, n_{0}=1, k_{0}=2, \bar{p}=3 / 4, M=40, N=8$,

$$
\begin{gathered}
a_{n}=(n+1)^{2} \ln ^{3}(n+2), \quad p_{n}=\frac{3 n-4}{4 n+2}, \quad d_{1 n}=\frac{n(1-n)}{2}, \quad d_{2 n}=\frac{n(3-n)}{2}, \\
g_{n}=\frac{(-1)^{n}}{n^{2}}, \quad f(n, u, v)=\frac{u^{2} v^{3}}{\sqrt{n^{3}+1}}, \quad \forall(n, u, v) \in \mathbb{N} \times \mathbb{R}^{2} .
\end{gathered}
$$

It is clear that (2.1), (2.2) and (2.33) hold. Consequently Theorem 2.4 guarantees that (3.3) has a bounded positive solution in $\overline{U(M)}$. But Theorem 2 in [11] is inapplicable for (3.3).

Example 3.3. Consider the third order neutral delay discrete equation

$$
\Delta\left(\sqrt{n^{5}+1} \Delta^{2}\left(x_{n}-\frac{n^{3}+1}{3 n^{3}+4} x_{n-\tau}\right)\right)+\frac{\sqrt{n} x_{n^{2}-2 n}^{6}}{n^{4}+n+1}+\frac{x_{2 n+3}^{3}}{n^{2}+2}=\frac{\sin \left(n^{2}-n\right)}{n^{2}+1}, \quad n \geq 1,
$$


where $\tau \in \mathbb{N}$ is fixed. Let $l=2, n_{0}=1, k_{0}=3, \underline{p}=1 / 3, M=30, N=19$,

$$
\begin{aligned}
& a_{n}=\sqrt{n^{5}+1}, \quad p_{n}=-\frac{n^{3}+1}{3 n^{3}+4}, \quad d_{1 n}=n(3-n), \quad d_{2 n}=-n-3, \\
& g_{n}=\frac{\sin \left(n^{2}-n\right)}{n^{2}+1}, \quad f(n, u, v)=\frac{u^{6} \sqrt{n}}{n^{4}+n+1}+\frac{v^{3}}{n^{2}+2}, \quad \forall(n, u, v) \in \mathbb{N} \times \mathbb{R}^{2} .
\end{aligned}
$$

Obviously, (2.1), (2.2) and (2.34) hold. Thus Theorem 2.5 ensures that (3.5) has a bounded positive solution in $\overline{U(M)}$. While Theorem 2 in [11] is unfit for (3.5)

\section{Acknowledgment}

The authors would like to thank the editor and referees for useful comments and suggestions. This study was supported by research funds from Dong-A University.

\section{References}

[1] R. P. Agarwal, S. R. Grace, and D. O'Regan, "Nonoscillatory solutions for discrete equations," Computers E Mathematics with Applications, vol. 45, no. 6-9, pp. 1297-1302, 2003.

[2] A. Andruch-Sobiło and M. Migda, "On the oscillation of solutions of third order linear difference equations of neutral type," Mathematica Bohemica, vol. 130, no. 1, pp. 19-33, 2005.

[3] J. Baštinec, L. Berezansky, J. Diblík, and Z. Šmarda, "A final result on the oscillation of solutions of the linear discrete delayed equation $\Delta x(n)=-p(n) x(n-k)$ with a positive coefficient," Abstract and Applied Analysis, vol. 2011, Article ID 586328, 28 pages, 2011.

[4] J. Baštinec and J. Diblík, "Subdominant positive solutions of the discrete equation $\Delta u(k+n)=$ - $p(k) u(k), "$ Abstract and Applied Analysis, vol. 2004, no. 6, pp. 461-470, 2004.

[5] J. Baštinec and J. Diblík, "Remark on positive solutions of discrete equation $\Delta u(k+n)=-p(k) u(k)$," Nonlinear Analysis, vol. 63, no. 5-7, pp. e2145-e2151, 2005.

[6] J. Baštinec, J. Diblík, and Z. Šmarda, "Existence of positive solutions of discrete linear equations with a single delay," Journal of Difference Equations and Applications, vol. 16, no. 9, pp. 1047-1056, 2010.

[7] J. F. Cheng and Y. M. Chu, "Oscillation theorem for second-order difference equations," Taiwanese Journal of Mathematics, vol. 12, no. 3, pp. 623-633, 2008.

[8] S. S. Cheng and W. T. Patula, "An existence theorem for a nonlinear difference equation," Nonlinear Analysis, vol. 20, no. 3, pp. 193-203, 1993.

[9] W. T. Li, X. L. Fan, and C. K. Zhong, "On unbounded positive solutions of second-order difference equations with a singular nonlinear term," Journal of Mathematical Analysis and Applications, vol. 246, no. 1 , pp. $80-88,2000$.

[10] M. Migda and J. Migda, "Asymtotic properties of solutions of second-order neutral difference equations," Nonlinear Analysis, vol. 63, pp. 789-799, 2005.

[11] J. Yan and B. Liu, "Asymptotic behavior of a nonlinear delay difference equation," Applied Mathematics Letters, vol. 8, pp. 1-5, 1995. 


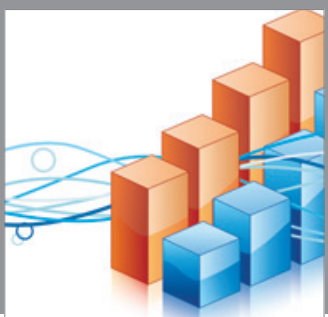

Advances in

Operations Research

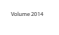

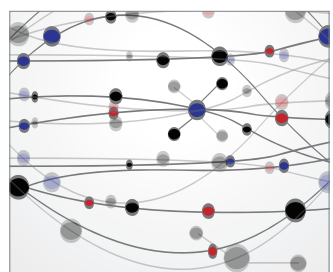

\section{The Scientific} World Journal
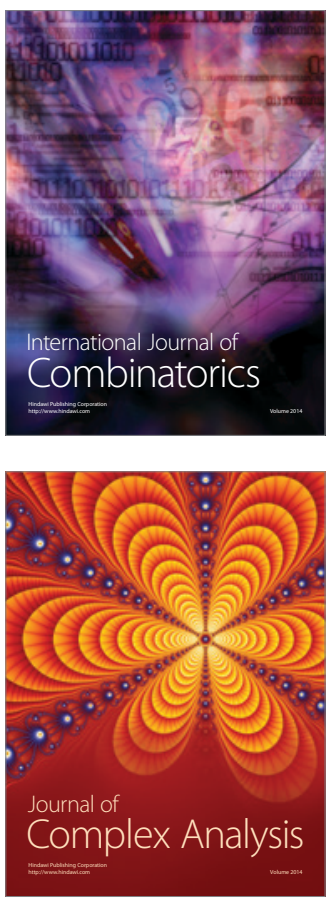

International Journal of

Mathematics and

Mathematical

Sciences
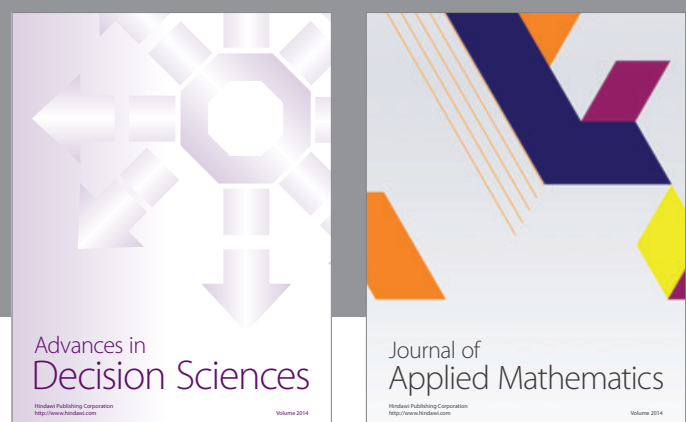

Journal of

Applied Mathematics
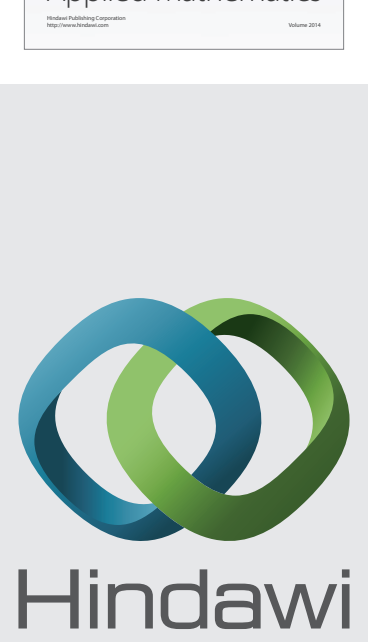

Submit your manuscripts at http://www.hindawi.com
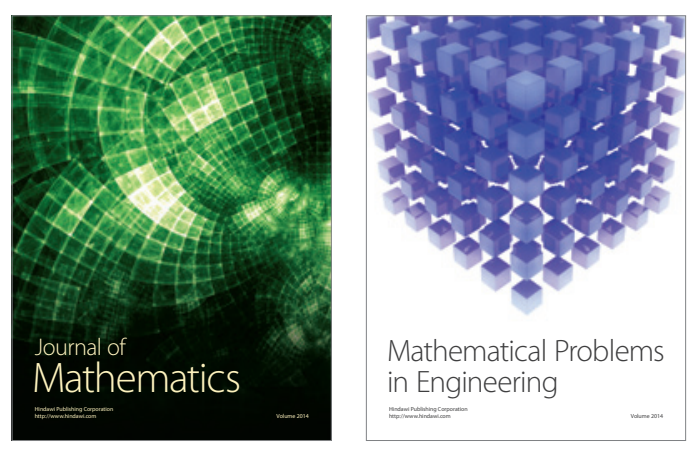

Mathematical Problems in Engineering
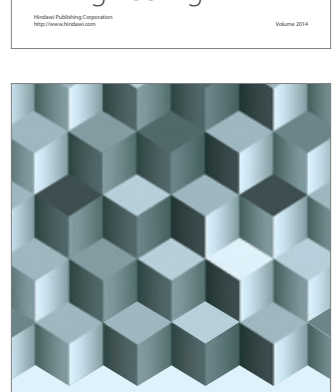

Journal of

Function Spaces
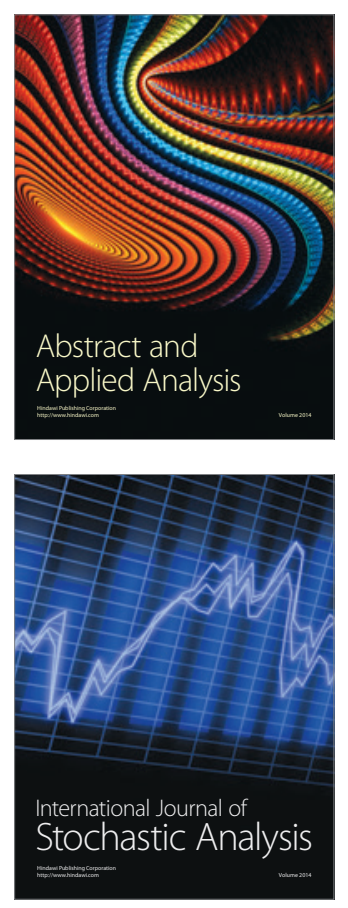

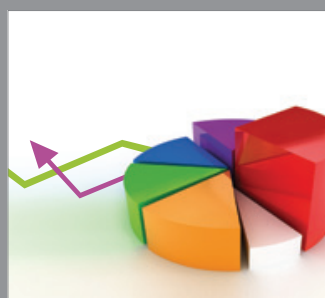

ournal of

Probability and Statistics

Promensencen
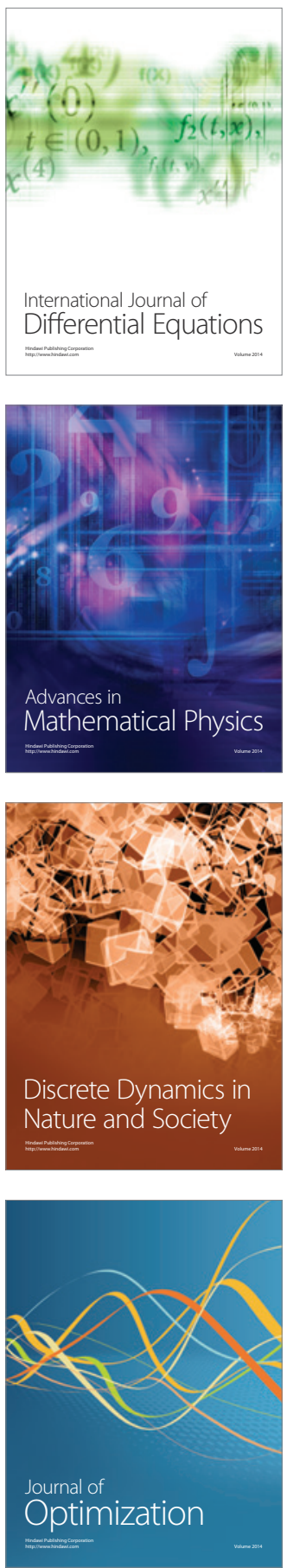